\title{
Combined Horizontal Split Gluteus Maximus Muscle Flap and Fasciocutaneous Limberg Flap for Reconstruction of Recurrent Sacrococcygeal Pilonidal Sinus
}

\author{
MAHFOUZ S.I. AHMAD, M.D., M.R.C.S. \\ The Department of Plastic Surgery, Faculty of Medicine, Zagazig University, Egypt
}

\begin{abstract}
Background: Pilonidal sinus is a chronic recurrent medical disease. The exact etiology of the disease is still unknown but the most accepted theory is an acquired condition characterized by infected sinus in the natal cleft area contains lifeless hair tuft. Multiple techniques were prescribed for its treatment however the ideal method still not yet defined.

Patients and Methods: Fifty eight male patients who underwent excision of their recurrent pilonidal sinus disease and the resulting defects were reconstructed using combined horizontal split gluteus maximus flaps and rhomboid flaps. Outcomes were revised from patient's files case and followed up in outpatient clinic and via questionnaires.
\end{abstract}

Results: The mean hospital stay was three days. The mean time to return to work was 16 days. Partial wound dehiscence's occurred in two patients. Distal end flap necrosis occurred in one patient. There were no flap losses, no recurrences, no infections, no loss of function and no seromas during a mean follow-up period of 24 months. All patients were satisfied with the results.

Conclusion: In comparison with other techniques, it has a comparable operative time and hospital stays. It has minimal and acceptable complication rates and no recurrences. We can conclude that this procedure of combined split gluteus maximus muscle flap and rhomboid flap provides an excellent, effective, easy and feasible method of choice for reconstructing defects of recurrent pilonidal sinus disease.

Key Words: Pilonidal sinus disease - Recurrent - Split gluteus maximus flap - Rhomboid flap.

\section{INTRODUCTION}

Pilonidal sinus (PNS) is a common recurrent chronic inflammatory disease. It affects about $0.7 \%$ of population [1]. It leads to work intolerance as it has a very high recurrence rate [2]. The exact etiology of the disease is still unknown. The most accepted theory postulated that PNS is an acquired condition characterized by infected sinus in the natal cleft area contains lifeless hair tuft [3]. There are multiple techniques prescribed for its treatment however the ideal method still not yet defined [4-11].

The use of rhomboid flap reconstruction of pilonidal sinus was first described by Azab et al., 1984 [12]. Since then many series have been performed to evaluate and compare it with other methods. It shows promising results however it still has a considerable incidence of recurrence and complication rates [13,14]. Even though many modifications of lateralization, superiorly based, oval head and/or perforator rhomboid have been performed, it still has a considerable incidence of complications and recurrences [15-17].

PNS wound is usually ischemic and present in a contaminated area so the use of muscle flaps carries a beneficial effect in these situations. Muscle flaps are very good options in reconstruction of such wounds because of their very rich blood supply and ability of obliteration of dead spaces. In addition to promoting rapid wound healing, it increases the ability of the wound to resist infections $[18,19]$.

This study presented a technique of combining two independent flaps in two layers for reconstruction of recurrent pilonidal sinus.

\section{PATIENTS AND METHODS}

Fifty eight male patients with recurrent pilonidal sinus presented to Plastic Surgery Department during the period from March 2016 to July 2019. The mean age was 23 years (ranged between 18 to 40 years). From history and revision of previous 
patient's files, forty one patients $(71 \%)$ were operated by excision and primary closure. Ten patients $(17 \%)$ were operated by excision and their wounds left opened for secondary healing. Four patients (7\%) were operated by Karydakis's procedure which is asymmetric closure technique with a straight edge incision down to the presacral fascia on one side and a sloping (45 degrees) edge on the other side [20]. Three patients (5\%) were operated by Bascom's procedure which is a closure technique in which asymmetrical elliptical incision is carried out around the midline wound into the subcutaneous fat to wedge out the wound then a dermal flap is undermined and closed [5].

Twenty eight patients (48.2\%) had more than one operation before admission at our department. All patients were consented for the procedure after it has been approved by the institutional review board. Doppler study was performed preoperatively for all patients to define the perforators of parasacral, superior and inferior gluteal vessels.

\section{Inclusion criteria:}

1- Patients who signed the informed consent.

2- Patients with recurrent pilonidal sinus.

3- Pilonidal sinus without of signs infection at time of surgery.

\section{Exclusion criteria:}

1- Pilonidal sinus with signs of infections.

2- Patients who refused to sign the consent.

3 - Non recurrent pilonidal sinus.

\section{Surgical procedure:}

All patients were operated under general anaesthesia. Patients were placed in prone jackknife position. All patients received a prophylactic single dose of 1.5 grams cefazoline intravenously at time of induction.

Adhesive tapes were used to retract buttocks laterally. Patient's lower back, buttocks and natal cleft was washed using povidone iodine solution $10 \%$. The affected area which will be removed was marked in the shape of rhombus that included all sinuses openings. According to the size of the resulting defect either single or double rhomboid fasciocutaneous flaps were designed (Figs. 1A,2B). Methylene blue was injected into all visible openings to delineate all sinuses tracts (Fig. 1B). The affected area then removed completely in block including all sinuses tracts and confirmed from methylene blue dye. The rhomboid flap/s were dissected and raised completely from the underlying gluteus maximus muscle fascia and hung with sutures. One or two split gluteus muscle flap/s (depending on the defect size) was then raised from lateral to medial keeping its medial attachment to preserve the perforators (Fig. 1C). After proper heamostasis was performed, the adhesive taps were removed to help tension free closure. The muscle flap was turned over upon itself 180 degrees and sutured over the presacral fascia using 4/0 polyglactin 910 sutures forming cushion like over the midline (Fig. 1D). The rhomboid flap/s were then mobilized and sutured to close the defect in two layers over a negative pressure hemovac drain. The skin was closed using 4/0 polypropylene sutures. Patients were then kept postoperatively in prone position during their hospital stays then after discharge they were allowed to change between prone and lateral positions during first two weeks postoperatively however sitting position was minimized. During the first postoperative day only oral fluids were allowed. Drains were removed between $2^{\text {nd }}$ and $4^{\text {th }}$ postoperative days and patients were discharged home. Patients's satisfactions were evaluated via answering the following questionnaire at six month and at one year after the procedure using modified Elalfy satisfaction score [10] (Fig. 3).

\section{RESULTS}

All 58 patients were operated upon by same procedure. The mean operative time was 65 minutes (ranged between 55 minutes to 78 minutes). Skin sutures were removed after two weeks. Patient's return to work was recorded. The patients were scheduled for follow-up at one month, two months, six months, and yearly thereafter. Sound healing was achieved in fifty five flaps without any complications (Figs. 1E,2B and 2C). Two patients (3.4\%) had partial wound dehiscence's which were managed by local wound care. Another patient $(1.7 \%)$ developed distal end flap necrosis which as managed by local debridement and re suturing. There were no flap losses, no recurrences, no infections and no seromas during a mean followup period of 24 months (ranged between 7 to 32 months). The mean time to return to work was 16 days (ranged from 14 to 21 days). The mean hospital stay was 3 days (ranged from 2 to 4 days). Results of patients satisfactions came as follow, about $94.8 \%$ of patients were highly satisfied while only $5.2 \%$ were satisfied. Patient's characteristics and their satisfaction with the procedure were summarized (Table 1). 


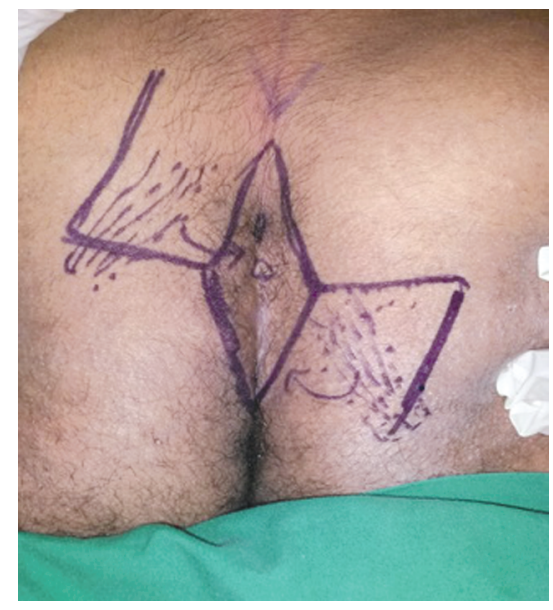

Fig. (1A): Perforators are detected and marked and two rhomboid flaps are outlined with dotted lines that marks the underlying muscle flaps.

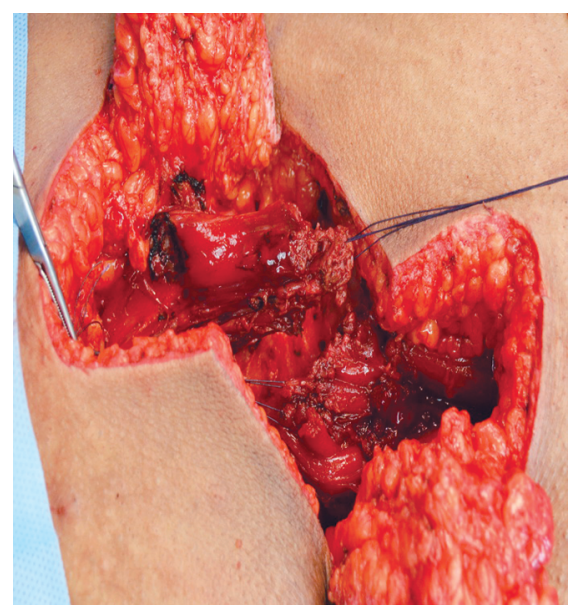

Fig. (1C): Intra operative view after preparation of two rhomboids and two horizontally split gluteus muscle flaps.

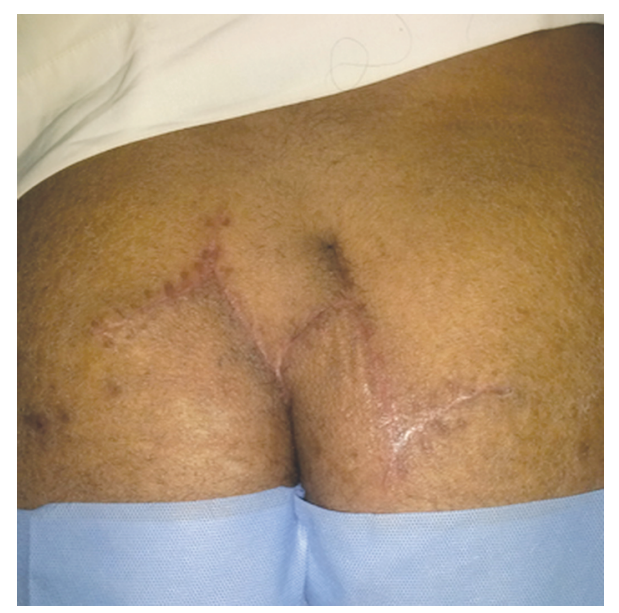

Fig. (1E): Postoperative view after one year, stable wound without recurrence.

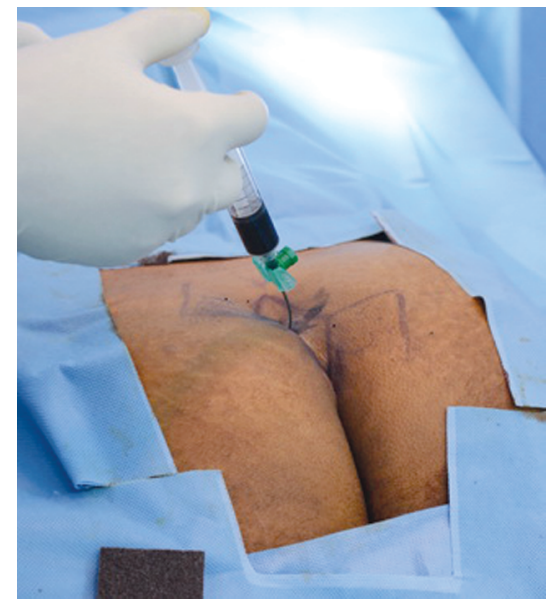

Fig. (1B): Injection of methylene blue into the sinus tracks.

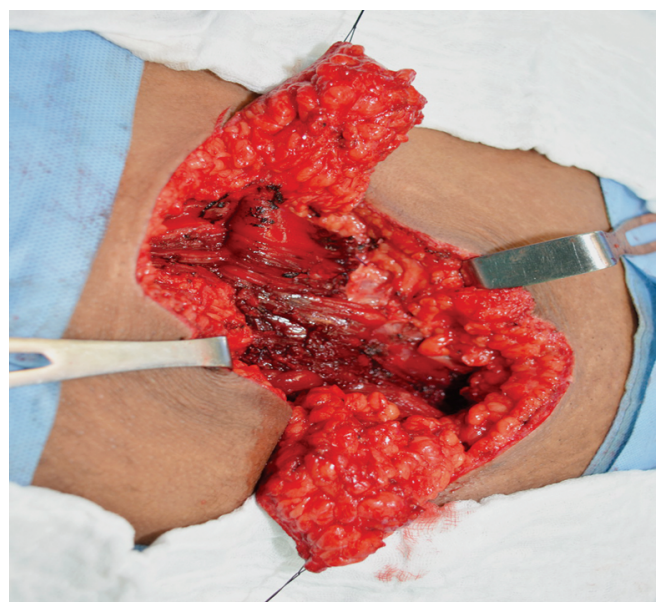

Fig. (1D): The partial split muscle flaps turned over 180 degrees and transposed into the defect.

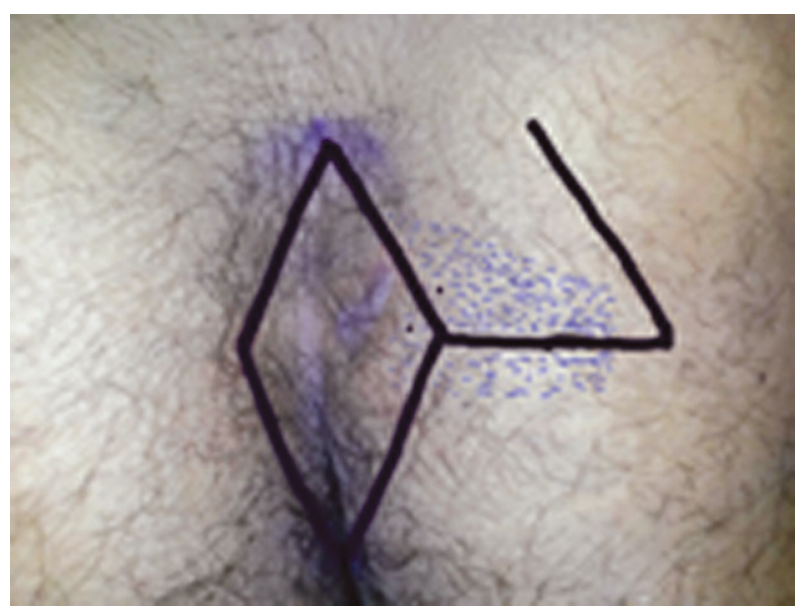

Fig. (2A): Preoperative design of the rhomboid flap and the rhombus design of excised area including all sinuses. Marking of perforator sites performed as identified by Doppler. Split muscle flap marked with dotted lines. 


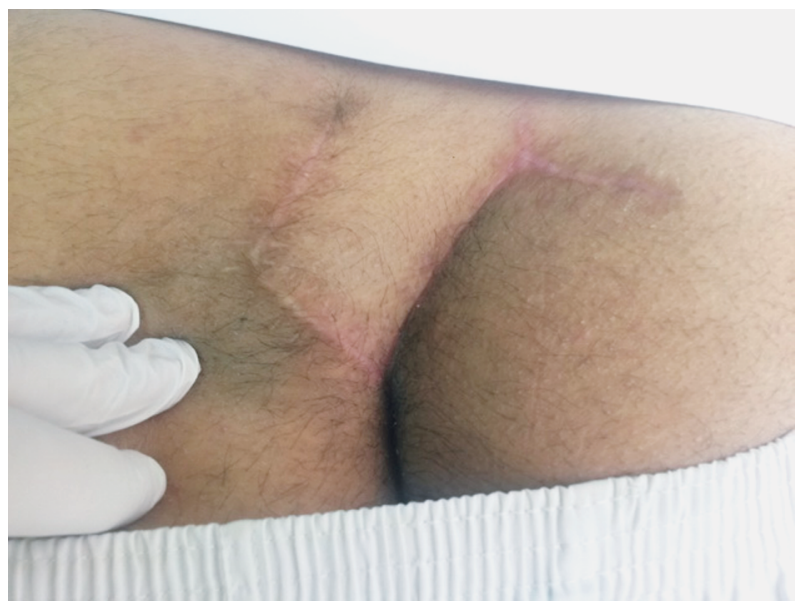

Fig. (2B): Post operative view after few months.

\begin{tabular}{lc}
\hline Question & $\begin{array}{c}\text { Given } \\
\text { score }\end{array}$ \\
\hline A- Are you satisfied with the cosmetic appearance? & \\
- Highly satisfied & 4 \\
- Satisfied & 3 \\
- Dissatisfied & 2 \\
- Highly dissatisfied & 1 \\
B- Have you required any further treatment? & \\
- No & 4 \\
- Yes currettage and dressing & 3 \\
- Yes drainage of an abscess & 2 \\
- Yes surgery for recurrence & 1 \\
C- When you resumed return to regular work? & \\
- After two weeks & 4 \\
- Within three weeks & 3 \\
- Within four weeks & 2 \\
- Afte more than four weeks & 1 \\
D- Would you recommend this technique to a friend? & \\
- Strongly recommend & 4 \\
- Fair & 3 \\
- Not recommend & 2 \\
- Strongly not recommend & 1 \\
E- Do you have any functional deficit in your hips? & \\
- No & 2 \\
- Yes & 1 \\
\hline
\end{tabular}

Fig. (3): Data collected, interpreted and score of 16 to 18 considered highly satisfied, score of 12 to 15 considered satisfied, score of seven to 11 considered dissatisfied and score of 6th or less considered strongly dissatisfied.

\section{DISCUSSION}

Pilonidal sinus disease is a common chronic inflammatory condition affecting young adults. Because of its high rate of recurrence, varieties of techniques have been prescribed [15].

The ideal reconstruction method for pilonidal sinus should be easy to be performed, leads to

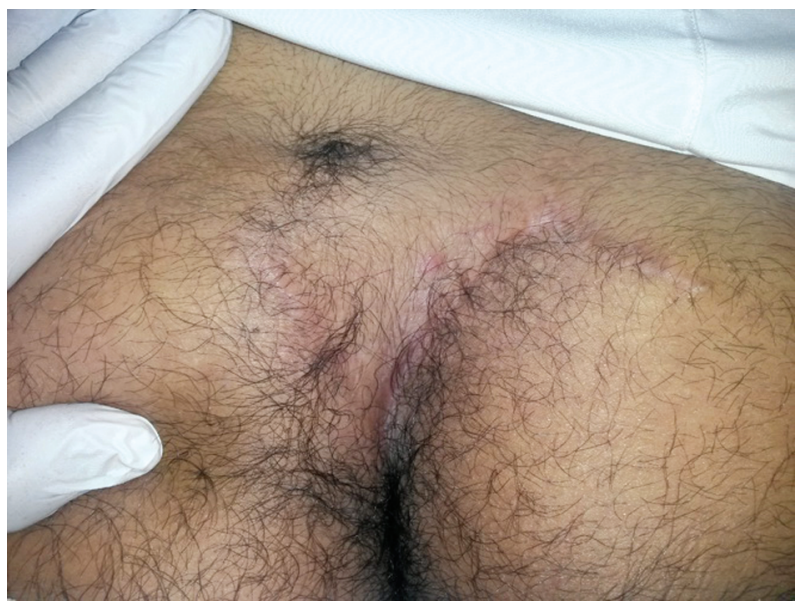

Fig. (2C): Late postoperative view (16 months).

Table (1): Patient's demographics, complications and patients satisfaction.

\begin{tabular}{lll}
\hline Item & $\begin{array}{c}\text { Mean } \\
\text { (range) }\end{array}$ & $\begin{array}{c}\text { Number } \\
\text { (percentage \%) }\end{array}$ \\
\hline Age (years) & $23(18-40)$ & \\
Operative time (minutes) & $65(55-78)$ & \\
Hospital stays (days) & $3(2-4)$ & \\
Complications: & & $2(3.4 \%)$ \\
$\quad$ Partial wound dehiscence & & $1(1.7 \%)$ \\
Distal flap necrosis & & 0 \\
$\quad$ Recurrence & & \\
Follow-up period & $24(7-32)$ & \\
Return to work (days) & $16(14-21)$ & \\
Patient's satisfaction: & & $55(94.8 \%)$ \\
$\quad$ Highly satisfied & & $3(5.2 \%)$ \\
$\quad$ Satisfied & & 0 \\
$\quad$ Dissatisfied and & & \\
strongly dissatisfied & & \\
\hline
\end{tabular}

complete excision of all sinus tracts, completely obliterates or flatten the natal cleft with a well vascularized tissue, provides neat healing, has a very low or no incidence of recurrence, has low complication rate, leads to rapid recovery and hence less hospital stay and quick resumption of patient's daily activities and return to work $[6,14,15]$.

Excision and primary closure have a high rate of recurrence (up to $30 \%$ ) and wound infections (about 7\%) and this because of the resulting midline wound with more pressure and tension $[15,16]$.

Wide excision and laying wound open to heal by secondary intention although it has lower incidence of recurrence $(10 \%)$ than primary closure $(30 \%)$, it requires a very long healing time with frequent dressings hence longer hospital stays, exhaustion of resources and human powers beside patient discomfort $[\mathbf{1 , 8 , 1 0 , 1 6 ]}$. 
Bascom method for treatment of pilonidal sinus has high incidence of recurrences ranged between 7.3 to $16 \%[5,21]$.

Many flap techniques have been prescribed for reconstructing pilonidal sinus defects (e.g., Karydakis, V-Y, Z plasty, rhomboid, perforator flaps and modified rhomboid). Although each flap has its own pros and cons, but all are still having a considerable incidences of recurrences and wound infections. However still there is no consensus about the proper ideal method $[\mathbf{8 , 1 1 , 1 3 - 1 6 , 2 0 ]}$.

After reviewing the literature, it was noticed that all previously mentioned flaps are fasciocutaneous ones and split gluteus muscle flaps were not addressed before for management of such chronic inflammatory disease.

Of all currently available fasciocutaneous flaps, Limberg flap has been considered that most feasible and preferable surgical technique as it is simple, requires short hospital stay, allows early patient return to work, easy wound management and very low incidence of complications $[\mathbf{1 3 , 1 4 , 1 6 , 1 7 ]}$.

Rhomboid flap although preferable by most plastic surgeon, it still has considerable rate of recurrences which ranged from $0.8-6 \%$ but it is considered less than other flap techniques $[\mathbf{5 , 1 6}]$.

The value of muscle flaps in resurfacing of chronically infected wounds are well established as it has a very rich blood supply, ability for obliteration of dead space, rapid wound healing and ability to resist infections $[\mathbf{1 8 , 1 9 ]}$.

The only reported trials for use of gluteus maximus flap for reconstruction of pilonidal sinus disease were done by Perez when he used it as a rotation myocutaneous flap for a case of multi recurrent pilonidal disease [22]. Rosen and Davidson also used Perez idea but on five patients and they reported no functional sequel with acceptable morbidity [23]. The split gluteus maximus flap as a muscle with split thickness skin graft or as a myocutaneous flap has been reported only for reconstruction of sacral ulcers [24,25].

Local split gluteal muscle flap has low donor site morbidity, so its use for reconstruction of pilonidal sinus defect causes no risk neither on function nor cosmoses. This idea has been provided in previous series but for reconstruction of sacral pressure ulcers [24,26,27].

The aim of this study was to take benefits of both muscle and rhomboid flaps independently. In addition to prescribing this combination and its technical aspects, the study proved its successful and encouraging results in reconstructing recurrent pilonidal sinus diseases defects.

In this study we used a split gluteus maximus muscle flap raised from its caudal end and turned up on itself 180 degrees. The muscle flap was then covered by unilateral or bilateral rhomboid fasciocutaneous local flap. In this method the muscle acts as a cushion to fill the gap created by excision of pilonidal sinus. In addition the muscle resists infections by increasing local area nutrition due to its rich perforators which form new numerous vascular plexuses [24].

The fasciocutaneous flap was used to add more strength to the closure and more padding which leaded to elimination of natal cleft. The rhomboid flap was chosen as it has many advantages in comparison to other fasciocutaneous flaps [14-16].

The advantages of this combined flap are that although it is consisted of two different independent flaps, the technique is easy and not time consuming. The used flaps are local ones and their dissections required minimal effort.

In this study the mean operative time was 65 minutes which is slightly longer than other series which reported 45, 58 and 54 minutes but for one flap surgery only not for two combined flaps as done in this study $[\mathbf{1 5}, \mathbf{1 6 , 2 8}]$ while Elkatib and Albasti reported longer operative time of 80 minutes [29]. The mean time return to work was 16 days which comparable to other series $[\mathbf{1 6 , 3 0 , 3 1 ]}$. The mean hospital stay in this study was three days which is either comparable to some studies [15-17] or shorter than others $[\mathbf{1 2 , 1 4 ]}$.

The results of this study revealed no recurrences during a mean follow-up period of 24 months. While two patients $(3.4 \%)$ developed partial wound dehiscence and one (1.7\%) developed distal flap necrosis.

In a study done by Topgul et al., on 200 patients operated by rhomboid flaps they reported $2.5 \%$ recurrence rate, $3 \%$ distal flap necrosis, $1.5 \%$ seroma and $1.5 \%$ wound infection [12]. Daphan reported $4.8 \%$ recurrence rate, $4.1 \%$ partial wound dehiscence and 2\% seroma in 147 patients operated by rhomboid flaps [13].

In a large study on 238 patients operated up on by modified limberg flap. It revealed $1.27 \%$ recurrence rate and $0.8 \%$ wound infection [14]. 
Regarding patient's satisfaction in this study, $94.8 \%$ of them were highly satisfied and $5.2 \%$ were satisfied. Elalfy et al., reported $95 \%$ of patients were satisfied and 5\% were dissatisfied while Omar et al., reported $14 \%$ dissatisfaction rate of rhomboid flap patients [10,29].

\section{Conclusion:}

In comparison with other techniques, this one has a comparable operative time, hospital stays and work resumption time while it has minimal and acceptable complication rates. There were no flap losses, no loss of function and no recurrences. We can conclude that this procedure of combined split gluteus maximus muscle flap and rhomboid flap provides an excellent, effective, easy and feasible method of choice for reconstructing defects of recurrent pilonidal sinus disease.

\section{REFERENCES}

1- Lim J. and Shabbir J.: Pilonidal sinus disease - A literature review. World J. Surg. Surgical Res., 1117: 1, 2019.

2- Harlak A., Mentes O., Kilic S., Coskun K., Duman K. and Yilmaz F.: Sacrococcygeal pilonidal disease: Analysis of previously proposed risk factors. Clinics (Sao Oaulo), 65: $125,2010$.

3- Duman K., Ozdemir Y. and Dandin O.: Pilonidal sinus disease - Etiological factors, pathogenesis and clinical features. Arch. Clin. Exp. Surg., 5: 228, 2016.

4- Yoldas K., Karaca C., Unalp O., Uguz A., Caliskan C., Akgun E. and Korkut M.: Recurrent pilonidal sinus: Lay open or flap closure, does it siffer?. Int. Surg., 98: 319, 2013.

5- Bascom J.U.: Repeat pilonidal operations. Am. J. Surg., 154: 118, 1987.

6- Elbanna H.G., Emile S.H., Youssef M., Thabet W., Abd El-Hamed T.M. and Ghnnam W.M.: Novel approach of treatment of pilonidal sinus disease with thrombin gelatin matrix as a sealant. Dis. Colon. Rectum., 59: 775, 2016.

7- Biter L.U., Beck G.M., Mannaerts G.H., Stok M.M., Ham A.C. and Grotenhuis B.A.: The use of negative-pressure wound therapy in pilonidal sinus disease: A randomized controlled trial comparing negative-pressure wound therapy versus standard open wound care after surgical excision. Dis. Colon. Rectum., 57: 1406, 2014.

8- Gurel N.S., Cu N.K. and Canbek S.: Elliptical rotation flap for pilonidal sinus. Am. J. Surg., 187: 300, 2004.

9- Venus M.R. and Titley O.G.: Outcomes in the repair of pilonidal sinus disease excision wounds using a parasacral perforator flap. Ann. R. Coll. Surg. engl., 94: 12, 2012.

10- Elalfy K., Emile S., Lotfy A., Youssef M. and Elfeki H.: Bilateral gluteal advancement flap for treatment of recurrent sacrococcygeal pilonidal disease: A prospective cohort study. Int. Sur., 29: 1, 2016.

11- Nelson R. and Lalonde D.: Treatment of the chronic pilonidal sinus wound with a local perforator-assisted transposition flap. Plast. Reconstr. Surg., 122: 47, 2008.
12- Azab A.S., Kamal M.S., Saad R.A., Aboual Ata K.A. and Ali N.A.: Radical cure of pilonidal sinus by a transposition Rhomboid flap. Br. J. Surg., 71: 154, 1984.

13- Topgül K., Ozdemir E., Kilic K., Gökbayir H. and Ferahkösse Z.: Long-term results of Limberg flap procedure for treatment of pilonidal sinus: A report of 200 cases. Dis. Colon. Rectum., 46: 1545, 2003.

14- Daphan C., Tekelioglu M.H. and Sayilgan C.: Limberg flap repair for pilonidal sinus disease. Dis. Colon. Rectum., 47: 233, 2004.

15- Mentes B.B., Leventoglu S., Cihan A., Tatliciouglu E., Akin M. and Oguz M.: Modified limberg transposition flap for sacrococcygeal pilonidal sinus. Surg. Today, 34: 419, 2004.

16- Abdou M., Darwish A. and Hassanin A.: Reconstruction following excision of sacrococcygeal pilonidal sinus with a perforator-based fasciocutaneous limberg flap. J. Plast. Reconstr. Aesthet., Surg., 63: 1176, 2010.

17- Saydam M., Ozturk O., Sinan H., Balta A.Z., Demir P., Ozer M.T. and Demirbas S.: Comparison of modified Limberg flap transposition and lateral advancement flap transposition with Burrow's triangle in the treatment of pilonidal sinus disease. Am. J. Surg., 210: 772, 2015.

18- Tan K.J., Lim C.T. and Lim Y.T.: The use of muscle flaps in the salvage of infected exposed implants for internal fixation. J. Bone Joint Surg., 92: 401, 2010.

19- Sakuraba M., Umezawa H., Miyamoto S. and Gulbins H.: Reconstructive surgery for bronchopleural fistula and empyema: New application of free fascial patch graft combined with free flap. Plast. Reconstr. Surg., 54 (3): $212,2017$.

20- Karydakis G.E.: Easy and successful treatment of pilonidal sinus after explanation of its causative processes. Aust. NZ. J. Surg., 62: 385, 1992.

21- Mosquera D.A. and Quayle J.B.: Bascom's operation for pilonidal sinus. J. R. Soc. Med., 88: 45, 1995.

22- Perez-Curri J.A., Temple W.J. and Ketcham A.S.: Gluteus maximus myocutaneous flap for the treatment of recalcitrant pilonidal disease. Dis. Colon. Rectum., 27: 262, 1984.

23- Rosen W. and Davidson J.S.: Gluteus maximus musculocutaneous flap for treatment of recalcitrant pilonidal disease. Ann. Plast. Surg., 37: 293, 1996.

24- Ali M.Z., Swati M.J. and Ali F.Z.: Split gluteus maximus turn-over flap for sacral pressure ulcer. Internet. J. Surg., 13: 1, 2007.

25- Roy N. and Varghese B.K.: Split gluteus maximus musculocutaneous islanded flap based on inferior gluteal artery for all sacral sore management: A new technique Intern. J. Scien. Res., 7: 1995, 2018.

26- Vincent P.L., Pinatel B., Viard R., Comparin J.P., Gir P. and Voulliaume D.: The gluteus maximus inferior splitmuscle flap for the cover of ischiatic pressure ulcers: Study of 61 cases. Ann. Chir. Plast. Esthet., 61: 845, 2016.

27- Weizhong L., Zheng Z. and Zuojun Z.: Application of split gluteus maximus muscle-adipofascial turnover flap and subcutaneous tension-reducing suture technique in repair of decubitus ulcers. Int. Surg., 99: 447, 2014. 
28- Acarturk T.O., Parsak C.K., Sakman G. and Demircan O.: Superior gluteal artery perforator flap in the reconstruction of pilonidal sinus. J. Plast. Reconstr. Aesthet. Surg., 63: 133, 2010.

29- El-Khatib H.A. and Al-Basti H.B.: A perforator-based bilobed fasciocutaneous flap: An additional tool for primary reconstruction following wide excision of sacrococcygeal pilonidal disease. J. Plast. Reconstr. Aesthet. Surg., 62: 494, 2009.
30- Omer Y., Hayrettin D., Murat C., Mustafa Y. and Evren D.D.: Comparison of modified limberg flap and modified elliptical rotation flap for pilonidal sinus surgery: A retrospective cohort study. Int. Surg., 16: 74, 2015.

31- Tokac M., Dumlul E.G., Aydin M.S., Yalcın A. and Kilic M.: Comparison of modified limberg flap and karydakis flap operations in pilonidal sinus surgery: Prospective randomized study. Int. Surg., 100: 870, 2015. 